\title{
Des soins éducatifs à l'éducation par le soin
}

\author{
Jean-Bernard Mauduit
}

\section{OpenEdition \\ Journals}

Édition électronique

URL : https://journals.openedition.org/leportique/878

DOI : 10.4000/leportique.878

ISSN : $1777-5280$

\section{Éditeur}

Association "Les Amis du Portique"

\section{Référence électronique}

Jean-Bernard Mauduit, « Des soins éducatifs à l'éducation par le soin », Le Portique [En ligne], e-

Portique, mis en ligne le 08 janvier 2007, consulté le 10 septembre 2022. URL : http://

journals.openedition.org/leportique/878; DOI : https://doi.org/10.4000/leportique.878

Ce document a été généré automatiquement le 10 septembre 2022.

Tous droits réservés 


\title{
Des soins éducatifs à l'éducation par le soin
}

\author{
Jean-Bernard Mauduit
}

1 Entre les soins et l'éducation, il y a un air de famille. La tâche d'éducateur est parfois décrite et pensée dans le langage de la médecine. On parle par exemple, à propos du métier d'éducateur, de carences, d'urgence sociale, de remédiation, de prévention. Inversement, le personnel soignant est parfois désigné par des termes de pédagogie, et on lui confie de fait des tâches d'éducation (Infirmières d'éducation). Kant, dans ses Réflexions sur l'éducation, range même les soins à l'intérieur de l'éducation, au sens des "précautions que les parents prennent pour éviter que les enfants ne fassent un usage nuisible de leurs forces $»^{1}$. Cette proximité ne doit pas masquer les différences fondamentales qui séparent ces deux secteurs de l'activité humaine. Les soins sont même, à certains égards, opposés à l'éducation, et c'est le but de la première partie de cet article de mettre en évidence cette opposition. Pourtant, si l'éducateur n'est pas celui qui soigne, l'éducation est inséparable des soins dans un autre sens, en ce qu'elle donne des tâches qu'il faut que l'éduqué soigne, en tant qu'elle éduque par le soin, thèse dont le développement fait l'objet de la deuxième partie.

Les soins : ce qu'on fait avec soin

2 Il faut, pour commencer, éviter de définir les soins de façon trop étroite. Les soins sont de toutes sortes: soins médicaux, très souvent, lorsque l'on parle, par exemple, de premiers soins, de soins d'urgence, de personnel soignant; on s'éloigne cependant de la médecine avec les soins dentaires, et on s'en détache tout à fait quand on vaque aux soins du ménage, quand on veille aux soins de propreté ou d'hygiène, quand on soigne ses invités, quand le musicien soigne ses tempi ou les nuances écrites sur la partition, quand l'éditeur soigne la typographie des ouvrages qu'il publie. Les soins d'une mère pour son enfant peuvent être assidus, vigilants, patients ; les mères ne sont pas pour autant des infirmières, car il ne s'agit pas particulièrement de la santé de l'enfant mais de tout ce qui lui est indispensable. Les soins sont « les actions par lesquelles on soigne quelqu'un ou quelque chose $»^{2}$ et soigner n'est spécialement ni panser les plaies ni administrer des perfusions. C'est, d'une façon générale, s'occuper correctement des personnes, des 
choses ou de l'exécution des tâches. S'en occuper, c'est-à-dire en prendre soin. Fait partie des soins tout ce que nous pouvons et devons faire et considérer avec soin.

Le soin est une modalité de l'action

Définir exactement ce que sont les soins conduit donc à s'interroger sur le soin. Le soin est une modalité de l'agir, non du pâtir. Ce qu'on subit, on ne le subit pas avec soin. On ne voit pas, on n'entend pas avec soin. A l'hôpital, le soin est l'exclusivité du personnel actif, médical ou non: on n'est pas malade avec soin, on n'est jamais un patient soigneux. Il faut évidemment suivre le traitement : on peut reprocher à un malade de ne pas obéir soigneusement aux prescriptions de son médecin, mais ce n'est pas au patient comme tel que le reproche s'adresse : c'est à celui qui doit se soigner, se faire l'agent des soins. On peut de même observer, regarder, écouter avec soin, mais le soigneux, dans cette affaire, relève de ce que l'expérience empirique a d'actif: on s'approche pour mieux voir, on change son angle de vision pour voir plus complètement, on se demande ce que l'on voit, on émet une hypothèse confrontée immédiatement à l'expérience qui l'infirme, la confirme ou la laisse incertaine. Tout cela relève de l'agir.

4 Tout agir cependant n'appelle pas le soin. Beaucoup de nos actions, plutôt que «sans soin ", expression qui suggère malencontreusement la négligence, sont « hors soin ", ne relèvent pas du soin, parce qu'elles n'appartiennent pas à ce qui appelle le soin. Certaines relèvent $d u$ machinal, se passent d'attention, or l'attention est une des composantes du soin. Une habitude peut être acquise avec soin, mais une fois prise, elle donne un aspect soigné aux choses exécutées « hors soin ». D’autre part, ce que nous faisons n'est pas toujours composé: nos oui et nos non récapitulent de longues affirmations et négations, présupposent de la réflexion, mais n'exigent aucun soin au moment de leur énonciation, car l'acquiescement comme la dénégation sont des instants, ils n'ont pas plus de durée que les départs ou les arrêts: ils arrêtent une longue maturation et débutent une nouvelle vie; ils résultent du soin, appellent de nouveaux soins, mais sont en eux-mêmes hors soin, de la même façon que les habitudes acquises. Est encore hors soin toute action qui se fait sous la poussée continuelle de l'envie, par exemple la lecture d'un livre captivant, une randonnée en montagne qui offre continuellement de nouveaux paysages fascinants. Ce qu'on fait par plaisir est hors soin : personne ne se promène soigneusement.

5 Les résistances de la langue sont, sur ces points, un excellent guide. Que nous apprennent-elles de positif sur le soin? Que le soin ne s'applique qu'aux actions composées d'opérations distinctes dont la synthèse s'effectue au fil du temps, qu'il se distingue du plaisir, qu'il implique l'attention. Le soin est appelé par ce qu'on nomme précisément en français une tâche. Une tâche appelle précisément ces trois dimensions du soin. Une tâche est un enchaînement d'opérations dirigé vers un terme et subordonné à une fin externe. Le traitement d'une carie dentaire, par exemple, exige une opération de diagnostic précis, une anesthésie locale, le nettoyage de la lésion, son rebouchage : chacune de ces opérations implique la précédente, comme condition de sa possibilité, et la suivante comme condition de l'achèvement de la tâche, une carie soignée, puis de l'atteinte de son but externe : la dent ne fait plus mal, sa fragilisation est suspendue. Tout cela s'échelonne dans le temps. Aucune tâche n'est instantanée ni homogène : certaines sont faciles mais aucune n'est simple. Pour des naufragés qui, du rivage d'une île déserte, aperçoivent un bateau, hurler une fois n'est pas une tâche ; appeler sur soi l'attention par des hurlements répétés, les remplacer ultérieurement 
par d'autres signaux, feu allumé, rayons solaires réfléchis par un bout de miroir et renvoyés dans la direction du bateau, et toute autre opération que l'échec des précédentes nécessitera, ou que leur succès permettra, tout cela constitue une tâche.

De la même façon, la tâche est indépendante du plaisir : on n'aime pas les tâches, on ne s'y livre pas par plaisir. Certaines sont indifférentes, beaucoup sont mornes, toutes sont entreprises par nécessité, même si cette nécessité est, en fin de compte, celle du plaisir. Le plaisir peut donner lieu à l'exécution de tâches : il y a des tâcherons du plaisir, qui préparent méthodiquement dans la journée le plaisir du soir, par exemple en cuisinant des heures ce qui sera mangé en quelques minutes. Cela ne change rien à ce fait que cuisiner soit sans plaisir, soit même, en certaines opérations, une corvée. Eplucher des légumes, des fruits, les couper en tranches, par exemple, est hors plaisir, comme le montre le fait qu'on cherche à s'en débarrasser en le confiant à des machines. L'invention de machines est un signe probant: on ne donne jamais aux machines ce qui fait plaisir et celui qui joue aux échecs avec plaisir peut, faute d'adversaire, laisser à l'ordinateur la partie adverse, mais jamais la sienne.

7 Une tâche exige de l'attention. Aucune n'est machinale, même si quelques opérations peuvent y être livrées à l'habitude. La tâche, en effet, n'est pas définie par une succession fixe de gestes, mais par une finalité interne, conceptuellement déterminée. Un lit fait est un lit fait. Mais si le terminus ad quem est connu et commun à tous les cas, le point de départ est variable: aucune nuit n'est semblable à une autre : certains dormeurs ont fait tomber leur oreiller, d'autres ont bavé dessus, certains dérangent à peine leur literie, certains autres débordent, froissent, mouillent de transpiration leurs draps. Faire les lits est donc une aventure multiforme, régulièrement surprenante, à laquelle aucune procédure uniforme ne peut être appliquée. Il faut donc adapter les gestes à chaque situation et, par conséquent, faire attention à eux au moins au moment d'en décider. Toute tâche implique cette obligation de réfléchir sur ce qu'on va devoir faire pour atteindre un terme qui n'appelle pas de réflexion. La définition univoque de ce terme est sans doute l'élément caractéristique de la tâche. Travailler à la tâche, c'est être rétribué pour un ouvrage achevé, par opposition au travail à l'heure, ou à la journée, qui rétribue une certaine quantité d'un travail laissé indéfini quant à son terme. On dit «du travail », comme "du sable », partitivement, à partir d'une quantité indéfinie et d'une masse informe. On peut "chercher du travail», mais la langue refuse qu'on cherche "de la tâche". C'est cette détermination du terme sans détermination complète des procédures qui exige l'investissement de l'attention. La tâche est donc le répondant exact du soin. Les soins, ce sont les tâches.

Soin et efficacité

8 Il y a, cela dit, une très grande majorité de tâches efficaces. Est-ce à dire que le soin équivaille à l'efficacité ? Les Romains répondaient très étrangement à cette question : Medicus curat, natura sanat, le médecin soigne, mais c'est la nature qui guérit. Non soigné, le malade serait très probablement mort, mais les soins médicaux ne suffisent pas à la guérison.. Ils y contribuent, ils y sont même nécessaires, sans être suffisants. La nature n'est ici qu'un nom, le nom de tout ce qui détermine la guérison en dehors de l'intervention humaine, de tout ce qui complète cette action. Celui dont on dit qu'il a été très bien soigné une fois sur deux est mort quand même.

9 C'est le paradoxe constitutif du soin, et des soins. Leur terme n'est pas leur but. Les soins ont en eux-mêmes leur finalité, ils cherchent leur propre achèvement et se replient sur lui en cas d'échec. Pourvus de la double finalité, externe et interne, ils 
visent la première mais ne maitrisent que la seconde. Ils trouvent donc moyen d'être irréprochables et parfaits au cœur même de l'échec, au milieu du désastre. Ceux qui soignent installent assez souvent l'excellence au sein de l'impuissance. L'inefficacité donne même, paradoxalement, la preuve du soin, car un succès trop rapide rend certains soins inutiles. Le soin ne donne toute sa mesure que lorsqu'on va au bout de la tâche, or il n'y a plus besoin d'y aller si l'objectif externe des soins, par exemple, en médecine, la guérison du patient, est atteint auparavant. Dans tous les cas contraires, la perfection des soins n'apparait qu'avec l'imperfection des traitements.

Le soigneux est modeste par essence. Il aide. «Aide-soignant » est une sorte de carré conceptuel : il aide celui qui aide. Les soins sont toujours de " petits soins ». Être « aux petits soins" avec quelqu'un, c'est le soigner, simplement. C'est faire tout ce qu'on peut, tout ce qu'on doit et tout ce qu'il faut, sans que cela suffise nécessairement. Il faut, cependant, qu'il y ait quelque fois suffisance : on n'apprend le nécessaire que par la double expérience de ce qui est toujours effectué et suffit ici mais ne réussit pas là.

11

\section{Soins et éducation}

C'est ce qui différencie les soins de l'éducation. L'éducation réussit. Sa finalité est purement externe. Nulle excellence immanente ne sauvera de son désastre l'éducateur dont les pupilles saccagent l'environnement, pillent, tuent et violent dans le voisinage de son institut ${ }^{3}$. L'enfant éduqué n'est pas celui qui a entendu dire qu'il ne fallait pas mentir, ni même celui qui en est convaincu: c'est celui qui s'abstient de mentir. L'éducation n'est pas une œuvre seulement, une tâche; c'est un résultat. Les grands éducateurs sont des génies, des inventeurs de procédés. Il arrive même qu'ils ne soient pas même des inventeurs, au sens strict. Un invention, en effet, se réutilise, est un objet joint à un procédé qui tombe, dès le début, dans le domaine commun, produit son effet et rend les services promis à quiconque l'utilise correctement, tandis que les procédés éducatifs inventés par les grands éducateurs ne produisent parfois de résultats qu'entre les mains de leur inventeur. Il n'y a pas eu deux Summerhill, et ceux qui se sont inspirés des méthodes de Neill, ou bien ont échoué ou ont eux-mêmes inventé autre chose. Summerhill est une sorte d'exploit individuel, dont on ne cesse pas de se demander comment il produisait ses résultats, le secret se trouvant quelque part dans la personnalité de son créateur. Les instituts géniaux disparaissent d'ailleurs souvent à la mort de leur créateur. Lorsque Neill décrit les « leçons particulières » qu'il donnait à Summerhill, qui sont de courtes séances de psychothérapie, il décrit l'opération comme une sorte d'improvisation allègre, dépourvue de "méthode préconçue $»^{4}$; l'important est qu'il y ait un résultat, parfois immédiat. Quand Makarenko, au début de l'existence de la « colonie Gorki », contre toutes les doctrines, au grand scandale de ses collaboratrices, et même au sien propre, envoie trois coups de poing successifs à la figure d'un jeune voyou qui le provoque et refuse d'obéir à une de ses directives, il ne soigne pas, il réagit brutalement, poussé par une «fureur sauvage $»^{5}$. Or ce geste improvisé est précisément le moment fondateur du travail éducatif : jusqu'alors, les pensionnaires ignoraient purement et simplement l'équipe des éducateurs quand ils ne se moquaient pas d'eux. Ils entraient et sortaient de la colonie comme ils voulaient, dévalisaient les villageois ; l'un d'entre eux avait même commis un meurtre. Aucune action éducative ne pouvait commencer. Ce que Makarenko appelle lui-même un "miracle $"^{6}$ est une improvisation, directement contraire à la légalité et à ce que préconisent tous ceux qu'il appelle «les pisseurs 
d'encre ", c'est-à-dire les théoriciens de la pédagogie, mais c'est une improvisation suivie d'effets.

13 L'éducateur ne cherche pas, ne tâtonne pas : il improvise, il invente et ses inventions agissent. Son action est à l'opposé des soins. Kant, au début de ses Réflexions sur l'éducation, définit les soins éducatifs comme les précautions à prendre pour empêcher l'enfant de faire un usage nuisible de ses forces. Il ajoute, par la suite, que toutes les inventions techniques destinées à empêcher l'enfant de se faire mal en tombant: maillot, lisières, roulettes, bourrelets, sont inutiles et même nocifs, parce qu'ils dissuadent ou empêchent l'enfant d'user de ses « instruments naturels ", c'est-à-dire de ses mains, et contrarient la vocation de l'homme, qui est d'apprendre librement à user de ses forces ${ }^{7}$. Ces "machines" sont précisément faites pour la commodité des éducateurs, leur épargnent d'avoir «à faire attention à ce que les enfants ne s'estropient pas $»^{8}$. Autrement dit, ils dispensent l'éducateur des soins. Plus l'éducateur invente, moins il soigne. Or l'éducateur est un inventeur, sinon de machines, au moins de dispositifs, de procédés efficaces ; l'éducateur est un créateur de machines éducatives, dont l'objectif est précisément d'éviter d'avoir à soigner, de la même façon que le métier à tisser automatique, inventé à l'époque de Rousseau et de Kant, dispense des soins du tissage manuel. Rousseau utilise dans l'Emile le terme d'instrument : « Il est bien étrange que depuis qu'on se mêle d'élever des enfants on n'ait imaginé d'autre instrument pour les conduire que l'émulation, la jalousie, l'envie, la vanité, l'avidité, la vile crainte, toutes les passions les plus dangereuses, etc. $»^{9}$ Un peu plus bas : «On a essayé tous les instruments hors un. Le seul précisément qui peut réussir ; la liberté bien réglée. ${ }^{10} »$

L'éducation est un travail sur l'être à éduquer, qui exige méthode, outils; impose à l'action éducative une rigueur pratique: faire ce qu'il faut pour obtenir ce que l'on veut. En elle, finalités interne et externe sont liées. Un travail bien fait rend le service cherché. Si ce qu'on a fabriqué ne fonctionne pas, c'est que le travail doit être revu et corrigé, a été mal fait. L'éducation, comme travail sur l'être à éduquer, débouche sur l'invention et sur l'usage d'instruments, outils et machines. Makarenko compare, à la fin du Poème pédagogique, l'éducation avec la mise en œuvre des matières premières, la résistance de ceux qu'on éduque avec celle de la matière qu'on met en forme :

Pourquoi, dans les établissements d'enseignement supérieur technique, étudionsnous la résistance des matériaux et dans les établissements d'enseignement supérieur pédagogique n'étudions-nous pas la résistance de la personnalité, quand on commence à l'éduquer? Et ce n'est pourtant un secret pour personne que cette résistance existe ${ }^{11}$.

Opposé à l'éducation, le soin se rapproche de l'enseignement avec lequel il partage son essentielle insuffisance. De la même façon que le médecin du proverbe latin soigne sans guérir, l'enseignant enseigne sans instruire. Le soin pédagogique qu'il met à préparer ses cours, à inventer ses exercices, à improviser ses questions et ses réponses, tout cela ne sert à rien tant que les élèves ne font rien pour l'écouter, le comprendre, tenir pour vrai ce qu'il avance et le mémoriser. L'enseignement est foncièrement inagissant : il s'adresse aux élèves mais ne fait rien d'eux, ni ne leur fait rien. La nature est au médecin ce que le travail des élèves est à l'enseignement. Il y a coopération dans l'une et l'autre tâche : entre le médecin et la «nature » comme entre le professeur et l'élève. Ceux de nos théoriciens de l'enseignement qui ne veulent plus le savoir aujourd'hui ramènent significativement l'enseignement à l'éducation. L'invention des situations didactiques remplace alors le soin pédagogique, l'efficience remplace l'attention à la 
tâche, comme les lisières et maillots dispensent les parents de surveiller leurs enfants. Dans les établissements scolaires, on diminue le nombre des surveillants; les professeurs sont vivement priés de cesser leurs admonestations. L'enseignement devient une formation, les études deviennent des apprentissages. J'ai essayé de montrer dans mon livre Le territoire de l'enseignant ${ }^{12}$ le caractère à la fois dominant et trompeur de ces représentations.

L'éducation par le soin

Si l'éducation ne soigne pas, tourne le dos aux soins, le soin, en revanche, éduque. Soigner une tâche, c'est d'abord la mener à terme, à bonne fin. C'est faire les choses « jusqu'au bout », ne pas les faire "à moitié ». Le prescrit est un ouvrage achevé, défini conceptuellement: un lit fait, une pièce aérée ou nettoyée. Un ménage fait soigneusement va au bout de la saleté ; il n'en laisse pas sous les meubles, au dessus des armoires. En particulier, il ne se contente pas de ce qui se voit. Le soin ne soigne pas les apparences, c'est-à-dire ce qu'on voit sans y faire attention, ce qui s'impose aux yeux, ce qui « saute aux yeux ». Le soin varie les points de vue; s'il nettoie, il va chercher la saleté où elle est. Il inspecte les lieux. Il se penche, s'agenouille, regarde sous les lits, grimpe sur un escabeau pour scruter le dessus des meubles, va voir derrière les rideaux. Il déplace ce qui peut masquer une saleté cachée : il retourne les paillassons, les tapis, les nappes, décroche les tableaux et les assiettes accrochées au mur. Il traque les miettes, les "moutons ». Il déplace les porte-parapluies, les machines à laver, les réfrigérateurs, découvre d'infâmes cernes, d'immondes auréoles, d'affreuses et anciennes croûtes. Il nettoie tout cela et remet en place méthodiquement les choses et meubles déplacés, de telle sorte qu'ils recouvrent maintenant aussi exactement le propre que naguère le sale. Le soin vise l'aspect, non l'apparence. Une pièce, une habitation, un jardin, une barbe, un costume ont un "aspect soigné » quand ils montrent qu'ils ont effectivement reçu les soins prescrits. L'aspect n'est pas l'apparence mais l'apparition de la réalité. L'aspect soigné est le mode d'apparition de l'effectivité de la tâche.

17 L'exhaustivité du soin comporte une dimension chronologique. Faire les choses en entier, c'est se poser la question de ce qui sera nécessaire demain et plus tard. Une réparation soigneuse supprime la panne mais supprime aussi ce qui pourrait la faire revenir. Le "bon Samaritain» de l'évangile ne soigne pas seulement les plaies présentes du blessé trouvé agonisant au bord de la route. Il s'occupe de l'avenir : il avance à l'aubergiste les frais des soins à donner ultérieurement. Il envisage la tâche dans son entier, il soigne l'avenir : il peut arriver des complications, il faudra refaire les pansements, une blessure peut se rouvrir, etc. De l'effectivité du soin, la durée de ses effets est l'indice. Comme l'oubli de l'avenir est un indice certain de négligence.

Cela dit, le soin n'épuise pas seulement le prescrit : il l'excède aussi. Il y a une façon négligente de s'en tenir au concept. Quand on dit à certains enfants de faire leur lit, ils bordent correctement les draps et la couverture, positionnent exactement édredons et oreillers, mais s'ils ont tiré le lit au milieu de la chambre pour pouvoir border commodément le côté de la literie qui se trouve normalement contre le mur, ils l'y laissent ensuite et répondent qu'on ne leur avait pas prescrit autre chose que de faire leur lit. La remise en ordre d'une pièce dont le nettoyage a rendu inévitable l'introduction d'un certain désordre, voilà un signe de soin qui ne renvoie pas au concept : le lit est parfaitement fait, la finalité interne de la tâche est atteinte. Faire le lit est autre chose que le ranger. Cependant on peut aussi considérer cette prescription, et 
probablement toute prescription, comme implicitement restrictive : il faut faire le lit, et rien d'autre. Or le désordre introduit par un nettoyage exhaustif est quelque chose d'autre, de la même façon qu'aérer une pièce en hiver apporte un air nouveau mais aussi une réfrigération qui peut être indésirable. Le strict a deux degrés : celui qui n'ajoute à la prescription que ce qui est techniquement nécessaire à son exécution, et celui qui, pour n'y ajouter absolument rien et s'y conformer en toute rigueur, annule ensuite ce qu'il a ajouté d'abord. Le premier degré du strict est donc théoriquement large et pratiquement restreint tandis que le second est théoriquement restreint et pratiquement large. Plus resserrée est la compréhension, plus ample est l'action. Faire la vaisselle avec soin, ce n'est pas seulement la faire en entier, mais aussi ajouter au lavage pur le nettoyage de ses effets secondaires, et l'effacement de ses conditions techniques; l'évier sali par l'eau de vaisselle, sali par ce qui nettoie, sera à son tour nettoyé, ainsi que les paillasses et les carrelages qui entourent l'évier; les résidus alimentaires bloqués dans les grilles d'écoulement seront évacués; les marques que l'eau de rinçage laisse sur les verres en séchant seront prévenues par l'essuyage ; les brosses, éponges, tampons à récurer seront rangés, c'est-à-dire ôtés de leur lieu d'usage de telle sorte que le nettoyage se nettoie, en quelque sorte, lui-même.

Dans ce qui est prescrit, dans toute tâche, quatre dimensions coexistent, qu'il faut penser séparément: une prescription d'objectif final à atteindre (finalité interne) : une pièce nettoyée, aérée, un lit fait ; une prescription (implicite) d'opérations spécifiques liées à cet objectif : pour une pièce nettoyée, les opérations de nettoyage proprement dit, balayer, laver, épousseter, aspirer, récurer, etc. Ces opérations spécifiques visent directement l'objectif; elles sont donc implicitement présentes dans la prescription d'objectif, et s'en déduisent immédiatement. Il faut ensuite considérer comme impliquées indirectement toutes les opérations étrangères à l'objectif proprement dit, qu'il faut cependant effectuer à titre de conditions indispensables, par exemple, si on nettoie, déplacer les meubles sous lesquels il est impossible de nettoyer, se procurer les instruments indispensables, les mettre en état de fonctionner (vider les sacs d'aspirateurs pleins, etc.). Tout cela fait l'objet de ce qu'on peut appeler prescription d'opérations conditionnantes. Il faut enfin tenir pour inhérent à l'esprit de la prescription, quoique non impliqué par son concept, qu'on n'avait que cet objectif, et aucun autre, à atteindre. Par conséquent, dans le cas du nettoyage, seule la propreté devait être ajoutée à l'état initial des lieux; les autres aspects devaient rester en l'état: l'emplacement premier des meubles, la température des pièces, la place des objets, etc. Tous les effets que les opérations conditionnantes ajoutent par force doivent donc, si on suit l'esprit de la tâche, être soumis à une prescription d'annulation des effets non prescrits, c'est-à-dire être annulés par les opérations inverses : dans le cas du ménage, remise des meubles et des objets à leur place initiale ; s'il s'agit d'aérer, restitution de la température initiale de la pièce.

Le soin mis à une tâche implique chacun de ces moments: les deux premiers, à l'évidence, intuitivement, au nom de la seule application de la prescription d'objectif, mais les deux derniers tout autant; leur absence signifie toujours l'absence de soin, la négligence. Les opérations conditionnantes s'imposent discursivement, comme ces accidents nécessaires dont Aristote admet l'existence au livre IV de la Métaphysique ${ }^{13}$. Les opérations d'annulation d'effets non prescrits relèvent d'abord de l'interprétation : il faut comprendre la prescription d'objectif dans son esprit, mais ce qui découle de cet esprit est à nouveau discursif, « logique ». 
Comme modalité de l'action, le soin concerne donc à la fois l'activité intellectuelle et l'activité physique. Celui qui soigne son action la suit intellectuellement : il a l'objectif final présent à l'esprit et saisit chaque opération spécifique comme impliquée par lui directement ou non, et comme impliquée par la suivante, s'il y a conditionnement, ou rendue indispensable par la précédente, s'il y a simple remplacement d'une opération inefficace par une nouvelle. Soigner sa tâche, c'est comprendre ce qu'on fait, au fur et à mesure des opérations, et beaucoup de négligences respirent plutôt l'inintelligence que la seule mauvaise volonté. L'intelligence de ce qu'on fait n'en est pas, pour autant, l'explication, la raison que l'on s'en rend à soi-même explicitement. Le geste suit le geste, mais il ne s'ajoute pas à lui ; il le suit comme ce qui s'ensuit. Il peut ne pas être pensé, mais c'est un geste pensant.

Comme modalité de l'activité intellectuelle et pratique, le soin prend une signification pédagogique. Cette modalité de l'action est en effet susceptible de devenir une disposition d'esprit et de corps qui s'acquiert et se stabilise, un habitus, ou mieux encore, une qualité, au sens où ce mot s'oppose à un défaut. A ce titre, son importance éducative est absolument capitale. Le soin n'est pas la routine: l'habitus acquis est d'abord intellectuel; c'est une surveillance, une vigilance logique, qui éprouve, geste après geste, l'implication du précédent par le suivant, la nécessité de l'enchaînement entier des opérations par rapport à l'objectif final, et la relation de cet objectif interne avec la finalité externe de la tâche. Le soin ne s'endort jamais. Il sait, par définition ce qu'il fait et où il va. Il est entièrement dévoué à ce qu'il entreprend et effectue. Le soin ne vise jamais le mal, même si on peut faire le mal avec soin. On peut par exemple préparer un homicide avec soin. L'homicide est un crime, mais l'exécuter avec soin, c'est l'exécuter bien, c'est-à-dire complètement et purement, parfaitement. La perfection que le soin vise n'est pas une perfection morale, mais le pur respect pour la loi morale est une disposition d'esprit partiellement identique au pur respect de ce qu'on effectue, qui est l'essence même du soin. Quand on soigne quelqu'un, et non plus quelque action, le respect de l'action, qui peut être condamnable, se change en un respect pour quelqu'un, ce qui permet de comprendre que le lexique $d u$ soin (soigneux, soigneusement, soigner sa diction) puisse servir à désigner des choses infâmes, tandis que celui des soins n'a presque jamais d'application infamante. Le soin, en effet, soigne seulement l'action, alors que les soins soignent toujours en même temps une personne et impliquent son respect. Si le soin peut à la rigueur passer pour une vertu, c'est parce que le respect des personnes est une disposition d'esprit voisine du respect de ce qu'on fait. Il y a une sorte de gratuité de l'action soigneuse, car sa finalité externe, quoique présente, est, dans le soin, ce dont on fait abstraction. Entre le « souci de bien faire » et " le souci de faire le bien ", entre l'action faite par respect du devoir et l'action faite par respect de l'action elle-même, il y a une connivence manifeste. Le rigorisme du soin répond dans une certaine mesure au rigorisme moral. C'est un rigorisme provisoire. En tant que tel, il peut constituer une initiation à la rigueur morale, au comportement choisi par pur respect du devoir, comme les Stoïciens l'avaient bien compris. Epictète, dans ses Entretiens, raconte avoir subi une véhémente réprimande le jour où, enfant, il avait mal résolu un exercice de logique donné par son maître Musonius Rufus. Je n'ai tout de même pas, il me semble, incendié le Capitole, s'indigne l'élève, à quoi le maître répond, en substance que, dans le cas présent, l'exercice, c'est le Capitole ${ }^{14}$. La négligence logique, l'inattention aux données d'un problème scolaire, sont des fautes morales. Le soin est probablement la qualité qu'exerce le mieux la logique mais toute tâche que l'éducateur donne à faire a quelque chose d'une leçon de morale, dans la 
mesure où le soin s'y mêle, et, pour autant que l'objectif interne en soit moralement inattaquable, c'est même une leçon de morale sans aucune restriction.

Le soin dans l'entre-deux

Le soin n'est cependant pas une vertu. Ce n'est pas l'habitude de faire le bien: on peut soigner un méfait. Le soin est moralement indifférent, puisqu'il s'enferme dans le fait de sa tâche et ne veille pas au droit, aux valeurs, à l'obligatoire, au permis, à l'interdit. Sa finalité reste immanente : la conscience soigneuse ne cherche pas à obéir à une règle externe ; sa règle est la tâche achevée, quelle que soit cette tâche. De son côté, le pur respect du devoir, constitutif de la conscience morale, réfère la tâche entière et chacune de ses opérations constitutives à une norme externe, transcendante. L'appel du devoir est absolument indépendant de l'appel de la tâche, et le contredit parfois. Le soin s'inscrit dans une seule dimension horizontale, purement linéaire. Le pont de la rivière Kwaï est, pour le général anglais, ce qu'il faut achever, mais rapporté au devoir, c'est une traittrise sur laquelle, pendant quelque temps, on ne s'interrogera pas. L'enfermement unilatéral de l'attention est le propre de la conscience soigneuse.

Unilatérale, cette conscience s'épargne en particulier les affres de toute évaluation analytique, l'examen critique de chaque moment de la tâche. La conscience morale doit, elle, diriger sa lumière sur chacun de ces moments, pour en fixer le prix. Chacun coûte donc le prix de la décision à prendre. Chaque pas, pour la conscience morale est, par conséquent, une nouvelle mise en marche. L'inertie du geste appelé par le geste, entraînant le geste, est une énergie acquise au commencement de toute tâche. On se met à la tâche, c'est-à-dire qu'une fois qu'on s'y est mis, on est pris par elle, on est porté par elle. Le conscience soigneuse dispense de la charge d'effort que la conscience morale impose à chaque opération constitutive d'une tâche. La justice des fins couvre l'injustice des moyens L'impitoyable lumière morale isole, elle, tous les moments distincts, interdit toute confusion, toute contraction. Ce qui n'est que moyen ne se laisse pas couvrir par sa fin. La conscience soigneuse n'est pas aveugle: elle est seulement inattentive. Cette inattention est sa vertu principale, car elle entraîne l'innocence. La conscience soigneuse est une bonne conscience, une conscience légère. Telle est la conscience non thétique de chaque phonème, quand on parle, occupé à la tâche de dire sa pensée. En revanche, la conscience morale a pour analogue, dans le registre linguistique, la lourde attention, scrupuleuse, avec laquelle on s'efforce de bien prononcer un phonème inhabituel d'une langue qu'on est en train d'apprendre. Pour utiliser une autre métaphore, il y a une sorte d'inertie inhérente aux tâches et au soin : le poids de la décision demande une énergie, mais la poursuite de la tâche n'exige plus cette énergie : l'appel du devoir, qu'il faut réécouter à chaque étape, est assourdi par l'appel de la tâche, et le poids de l'obligation à mettre en œuvre s'allège, remplacé par l'entrain. Ce qu'il me faut faire maintenant n'est plus ce que je dois et ce que je décide, mais ce que le texte de la tâche commencée m'impose. La tâche entraîne celui qui s'y est mis. Chaque étape est donc envisagée autrement que comme ce qui engage la responsabilité et en fait sentir le poids, parfois cruel.

Le soin n'est donc pas seulement une loi, c'est un mobile. Il a une fonction d'économie énergétique. Soigner sa tâche, c'est s'écouter dicter par elle ce qu'il faut maintenant faire. Nulle lourde décision à prendre, nulle énergie nouvelle, autre que musculaire, à trouver et à investir. La tâche commande, et non plus la loi. L'autorité extérieure, non pas seulement celle de la loi morale, mais encore celles des autorités, recule et s'allège. La nécessité technique remplace, pour un temps, l'obligation légale, le commandement. 
Lorsque les pensionnaires de la colonie Gorki ont senti pour la première fois la colère du directeur et le poids de son autorité légale peser nettement sur eux, Makarenko les emmène couper des arbres en forêt.

A ma surprise, tout se passa à merveille. Je travaillai avec eux jusqu'au repas. Nous abattîmes les pins déjetés dans la forêt. Les gars étaient renfrognés en somme, mais l'air pur et glacial, la beauté des bois, aux arbres couverts de lourdes chapes de neige, le gai concert des scies et des haches, firent leur effet ${ }^{15}$.

Texte extrêmement parlant, quoique muet sur l'essentiel : la pacification des esprits provient de la tâche elle-même. On peut ne pas aimer ce qu'on fait, le faire avec mauvaise humeur, mais se mettre à la tâche, c'est sortir de la confrontation d'homme à homme, c'est échapper aux avatars de la relation d'autorité, car la tâche égalise et montre, par son avancement même, si ce qui se fait est bien fait. Le soin est un mobile ; il n'est nullement gratuit : on tient à terminer sa tâche; tout ce qui l'empêche est donc vécu comme frustrant. Ce n'est donc pas une affaire de pure volonté, mais qu'il mobilise n'en fait pas un désir non plus. On se promène par désir, mais on ne se promène pas avec soin. Le désir est une impatience du plaisir, mais aucune tâche ne produit directement de plaisir. Le plaisir advient, mais le soin est ce qu'on met à quelque chose, ou ce qu'on prend de quelque chose ou de quelqu'un. Le plaisir est une fin, ce qui fait plaisir est un moyen, mais aucune opération partielle n'est un moyen dont la suivante serait la fin. Le soin concerne ces impératifs hypothétiques qui ne sont cependant pas réductibles à l'adage "Qui veut la fin, veut les moyens. ». Le rapport du moyen à la fin ne concerne pas les opérations qui définissent une tâche. Même les opérations partielles considérées dans leur ensemble ne peuvent être considérées comme le moyen de l'achèvement de la tâche. Répandre le ballast d'une voie ferrée en construction n'est pas le moyen qui permettrait d'atteindre un but qui serait la pose des rails. C'est une condition technique. De la même façon, les travaux de terrassements, l'édification des ouvrages d'art, la pose des rails et l'électrification de la ligne ferroviaire ne constituent pas, en bloc, les moyens de l'achèvement du chantier, mais en sont les éléments constitutifs. La véritable fin est ailleurs : c'est l'effet supposé heureux de l'ouvrage achevé sur les conditions de vie des hommes concernés. Il faut sortir du chantier pour découvrir de véritables moyens. Les moyens sont toujours liés à une finalité externe. Cette finalité est toujours subjective, car elle implique une appréciation de sujets qui rapportent à eux ce qu'on met à leur disposition. Le plaisir est donc bien une fin, dont ce qui fait plaisir est le moyen. Le soin, qui envisage principalement l'opération partielle dans sa relation avec les autres et avec le tout de la tâche n'a donc aucun rapport ni avec le plaisir ni avec le désir. On peut, sans désir, tenir à ce qu'on fait.

27 Ni respect moral du devoir, ni désir, le soin nous apprend qu'il y a, entre le désir et la volonté, un espace intermédiaire, un entre-deux. La pure volonté se décide par pur respect du devoir, bon gré mal gré. Le pur désir obéit à la pression du plaisir attendu, connu ou au moins pressenti. Le soin se prend de ce qu'on entreprend et que l'on tient à poursuivre et à terminer. La tension existe, mais ce n'est ni celle de l'attente du plaisir, de son guet, ni l'insatisfaction qui résulte de l'imparfaite, incomplète ou insuffisante application du devoir. Ce vers quoi tend le soin n'est pas extérieur à la tâche, n'est ni une règle idéale ni une fin sensible : c'est ce qui achève la tâche, c'est la tâche ellemême comme achevée. Le soin tend à achever la tâche mais ne le désire ni ne le veut. obligation, qui soulève immédiatement l'objection du caractère indépendant de la 
volonté de l'amour. On peut bien s'obliger au respect, au devoir, mais on ne peut pas aimer par obligation. Kant aborde cette difficulté de la philosophie morale dans plusieurs de ses oeuvres, par exemple dans la première section des Fondements de la métaphysique des moeurs:

L'amour comme inclination ne peut pas se commander, mais faire le bien précisément par devoir, alors qu'il n'y a pas d'inclination pour nous y pousser, et même qu'une aversion naturelle et invincible s'y oppose, c'est là un amour pratique et non pathologique, qui réside dans la volonté et non dans le penchant de la sensibilité, dans les principes de l'action, et non dans une compassion amollissante ; or cet amour est le seul qui puisse être commandé. ${ }^{16}$

Pour sortir de la contradiction, Kant réduit donc l'amour du prochain au devoir de bienfaisance. Cette réduction est indéfendable: le devoir d'amour concerne le sentiment, il ne s'agit pas seulement de l'action. Les langues de la Bible ont probablement toute capacité de dire le devoir comme simple obligation d'agir. L'obligation d'amour oblige le sentiment. On ne peut certes pas obliger à être amoureux, ni même à être l'ami de quelqu'un, mais on peut obliger à cette disposition de l'intellect et du sentiment qu'est le soin. Ce qui est alors obligatoire dépend bien de la volonté : c'est l'attention apportée à tout ce que comporte l'achèvement d'une tâche entreprise pour quelqu'un. Le cœur est concerné, comme cœur qu'on met à l'ouvrage, comme sensibilité à ce qui, dans toute tâche, appelle son propre achèvement. On est sensible à cet appel, qui n'est ni celui du devoir ni celui du plaisir, mais celui du soin. L'amour du prochain est l'amour de celui que je traite avec soin, et c'est d'ailleurs ce qui fait de lui mon prochain, comme l'indique précisément la parabole évangélique du bon Samaritain.

Là se trouve le lien solide de l'éducation avec le soin. Eduquer n'est pas soigner, il n'existe pas de soins éducatifs. L'éducateur n'est pas à la place de l'infirmier, mais à celle de l'ingénieur, qui tire de ce qu'il sait, ou croit savoir, des lois du comportement enfantin, les procédés qui sont censés produire les transformations éducatives. L'efficacité est la règle de l'action éducative. Le soin n'intéresse l'éducation qu'à titre de procédé efficace, comme soin mis par celui qu'on éduque aux tâches qu'on lui fixe. Ce soin est une façon d'oeuvrer, d'abord intellectuellement, dont on peut prendre l'habitude et à laquelle l'éducateur peut exercer ceux qu'il éduque. Exercer un enfant ou un adolescent à envisager tout ce que comporte une tâche et ce qui peut l'excéder, puis à effectuer toute la tâche et seulement elle, c'est l'initier au soin. Cette initiation n'obéit pas au seul principe, ne s'effectue pas par pur respect du devoir : elle table sur un sens de la tâche, qui est une sensibilité à ce que la tâche elle-même appelle: son achèvement. Il y a donc une mobilisation affective induite par le seul fait de se mettre à la tâche, qui peut exiger l'effort d'une décision purement volontaire, mais se poursuit sur la lancée de cet effort, en suivant les "rails » de la tâche. Cette mobilisation n'est pas de l'ordre du désir. S'exercer au soin, c'est apprendre à s'intér-esser à la tâche, c'està-dire, étymologiquement, à occuper l'espace qui se trouve entre son commencement et son terme, à s'y enfermer. L'intérêt, pris en ce sens, est ce qui fait du soin un mobile : ce que je fais avec soin, je m'y intéresse. 


\section{NOTES}

1. Kant, Réflexions sur l'éducation Vrin, p.69.

2. Le grand Robert de la langue française, Dictionnaires Le Robert, 2001, tome 6, p. 515.

3. C'est la situation dans laquelle se trouve Makarenko quand il commence à diriger la « colonie Gorki », dont il ne se sauve d'abord qu'en cachant la vérité aux autorités. Cf. Poème pédagogique, Editions en langues étrangères, Moscou, 1953, pp. 34-39.

4. Neill, Libres enfants de Summerhill, Maspero Paris, 1975, p. 53.

5. Poème pédagogique, p. 39.

6. Ibidem, p. 39.

7. Kant, Ibidem, p. 99.

8. Ibidem, p.94.

9. Rousseau, Emile, Oeuvres complètes IV, Bibliothèque de la pléiade, p. 321.

10. Ibidem.

11. Makarenko, Poème pédagogique, Moscou, Editions en langues étrangères, 1953, p. 727.

12. J.B. Mauduit, Le territoire de l'enseignant, Paris, Klincksieck, 2003.

13. Aristote, Métaphysique, Livre "delta" ( livre IV).

14. Cf. Epictète, Entretiens I, VII, 32, in Les Stoïciens, Bibliothèque de la pléiade, Paris, Gallimard, 1962, p. 827.

15. Poème pédagogique p.40.

16. Kant, Fondements de la métaphysique des moeurs, Bibliothèque de la pléiade II, Paris, Gallimard, 1985, p.258

\section{RÉSUMÉS}

Eduquer ressemble superficiellement à soigner mais en diffère profondément parce que l'éducation se pense comme une pratique efficace, qui invente et utilise des instruments et des procédés supposés lui permettre d'atteindre son but, tandis que les soins, indépendamment de leur finalité externe, visent leur propre perfection d'abord et se concentrent donc sur une finalité interne. On peut mourir après avoir été très bien soigné. L'éducation ne soigne donc pas, mais le soin ne lui est pas étranger, dans la mesure où il s'agit d'une pratique intellectuelle et corporelle qui veille, à partir de la prescription d'une tâche, à son achèvement exhaustif et strict et peut donc être exercée par l'éducation et devenir un habitus. Intermédiaire entre désir et volonté, le soin n'est pas gratuit, car il repose sur l'intérêt.

Bringing up superficially amounts to providing care, but significantly differs from it, because bringing up defines itself as an efficient pactice which invents and uses instruments and procedures that are supposed to enable it to reach its objectives, whereas care, leaving aside its external finality, first and foremost aims at its own perfection and thus focuses on an internal finality. One may die after being very well taken care of. Therefore bringing up is not care, but care is not foreign to it, in so far as it is an intellectual and corporal approach which ensures the comprehensive and strict achievement of a task which had been previously prescribed. This 
specific practice can be carried out through bringing up and become a habitus. Somewhere between desire and willpower, caring is not gratuitous as it is based on interest.

Educar se parece superficialmente a cuidar, pero difiere profundamente de esto porque la educaci'on se piensa como una practica eficaz, que inventa y utiliza instrumentos y procedimientos que se supone le permiten lograr su fin, mientras que los cuidados, independientemente de su finalidad externa, sobretudo aspiran a su propria perfeccion y por lo tanto, se concentran es su finalidad interna. Se puede morir despues de haber sido muy bien cuidado. Por consecuencia, la educacion no cuida, pero de igual forma no es ajena a esto, en la media en que se trata de una practica intelectual y corporal que vela, a partir de la prescripcion de una tarea, por su cumplimiento exhaustivo y estricto y puede ser ejecido por la educacion y volverse un habito. El cuidado, intermediario entre deseo y voluntad, no es gratuito debido a que se apoya sobre el interès.

\section{AUTEUR}

\section{JEAN-BERNARD MAUDUIT}

L'auteur de cet article est professeur de lycée et a été chargé de cours à l'Université de Metz de 2000 à 2005. Il a mené une réflexion sur l'enseignement et sur l'éducation dans plusieurs articles, notamment « Enseignement, éducation et démocratie », dans le $n^{\circ} 29$ de la revue Le Télémaque, paru en mai 2006, et dans un livre Le territoire de l'enseignant, publié en 2003 chez Klincksieck. 